\title{
Polymerase Chain Reaction Thermoelectric Module Based on Prediction and Attachment of Aging Phenomena
}

\author{
Jong-Dae Kim, ${ }^{1,2}$ Jeong-Uk Park, ${ }^{1}$ Hye-Jeong Song, ${ }^{1,2}$ \\ Yu-Seop Kim, ${ }^{1,2}$ and Chan-Young Park ${ }^{1,2^{*}}$ \\ ${ }^{1}$ Department of Convergence Software, Hallym University, \\ 1, Hallimdaehak-gil, Chuncheon-si, Gangwon-do 24252, Republic of Korea \\ ${ }^{2}$ Bio-IT Research Center, Hallym University, \\ 1, Hallimdaehak-gil, Chuncheon-si, Gangwon-do 24252, Republic of Korea
}

(Received May 31, 2018; accepted November 22, 2018)

Keywords: polymerase chain reaction (PCR), machine aging, deep learning, machine learning

Many machines around us continue to age over time. This aging of machines affects experimental results, and the accuracy of experimental results may be reduced or not fully achieved. To prevent this problem, we decided to predict the lifetime of a machine to help us analyze experimental results. Thus, we carried out a polymerase chain reaction (PCR) experiment, which is an experiment to amplify the gene information stored in DNA to the amount required for gene analysis experiments, to determine the temperature change of a thermoelectric module (Peltier). The Peltier was used to determine the reliability of the results by learning how it affects the lifetime of the device. As a method of measuring aging, we used deep learning technology. In this study, we expect to learn how machines age. By learning the machine's own aging data of important parts, the reliability of test results can be greatly improved.

\section{Introduction}

Many types of devices around us age gradually as they continue to be used over time and behave differently from their intended use. Experimental instruments also age and behave differently from what is expected, so experiments are conducted in a direction different from the planned one. ${ }^{(1)}$ In addition, errors are observed in many results. This issue is so important that it has been discussed frequently.

Continuously, we investigate the polymerase chain reaction (PCR) instrument used for gene amplification experiments. PCR is a technique used to amplify a small amount of genes in DNA to enable DNA analysis experiments. ${ }^{(2-6)}$ This technique has a considerable effect on experiments, so the malfunctioning of the device will cause severe financial damage, longterm damage, and mispredictions. Subsequently, incorrect experimental results create the possibility of causing more problems in the analytical process. Therefore, we aim to prevent the occurrence of major problems in the experimental results owing to the aging of the equipment.

*Corresponding author: e-mail: cypark@hallym.ac.kr https://doi.org/10.18494/SAM.2019.2100 
As an alternative solution to the problem, we have determined that the use of machine learning, which has recently become a hot topic, can be an effective method of predicting the aging of the instruments themselves and the accuracy of the experiment. ${ }^{(7)}$ Thus, we have accumulated data of the experimental instrument over a long time. We learned about the deep learning structure, and thus, we can determine the degree of senescence. In instrument learning, we wanted to choose an application program interface (API) as simple and intuitive as possible. Finally, we conducted data analysis based on Keras, which is a high-level neural network API, written in Python. ${ }^{(8)}$

The PCR can be considered as a system with sensors and actuators. We use temperature sensors (NTC thermistor in this system) as sensors and the Peltier as an actuator. Thus, the results of this study can be applied to any sensor and actuator system.

In this paper, we present an index that predicts the degree of aging of a device in advance before analyzing the experimental results..$^{(7,9,10)}$ Such an index also predicts the result of a correct experiment through a normal device. We expect that Keras and other machine learning methods will greatly contribute to improving the accuracy of many experiments using machines.

\section{Materials and Methods}

First, the degree of aging of the PCR device is predicted. ${ }^{(2)}$ PCR basically controls the temperature through the Peltier effect using a thermoelectric module. ${ }^{(11)}$ Therefore, the most important factor in PCR instrument aging is how well the Peltier module predicts the degree of aging.

\subsection{Structure of PCR device}

Figure 1 shows the small commercially available PCR device used in the experiment (called MyPCR $\left.{ }^{\mathrm{TM}}\right) .{ }^{(12)}$ The PCR device is equipped with a thermoelectric module that controls the temperature internally and consists of a small amount of memory and a small number of components to run the program to control it.

In Fig. 2, we briefly show the PCR routine. First, timer 1 sends the temperature protocol to the PCR device for the experiment. The log of the recording starts from the beginning of the transmission and continues until the program ends. After receiving the experimental temperature protocol, the PCR device stores the protocol in its internal memory. Thereafter, the PCR device operates in accordance with the stored protocol, repeating the raising and lowering of the temperature. Finally, the process is terminated. As a result, we create a log file that contains the complete state of the experiment and an overall record of the experiment, and we use this file several times.

We can predict the degree of aging of the PCR device. PCR basically controls the temperature through the Peltier effect using a thermoelectric module. Therefore, the most important factor in PCR instrument aging is how well the Peltier module predicts the degree of aging. A brief description of a thermocycler indicates the use of a phenomenon in which a 
(a)

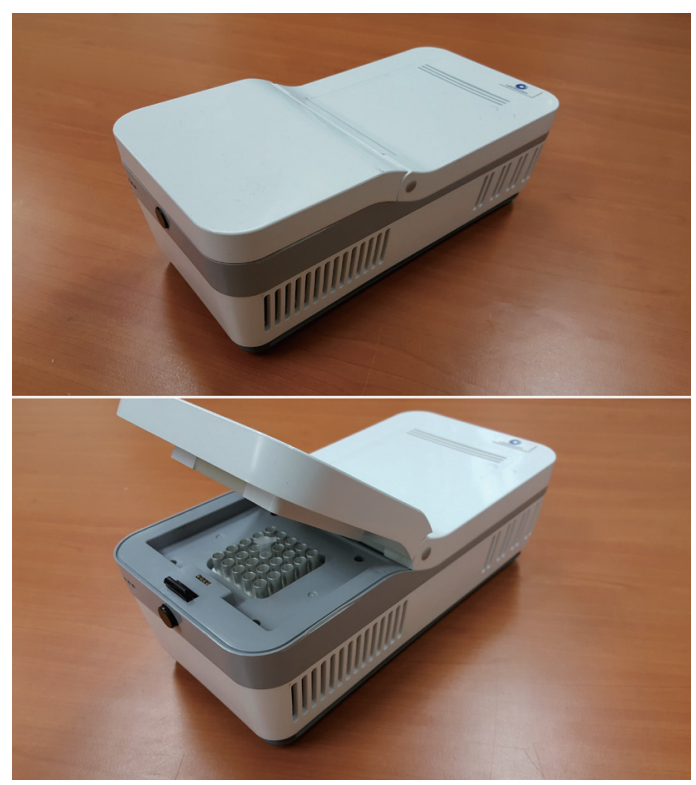

Fig. 1. (Color online) Exterior structure of PCR device. The lid is closed (a) and raised (b) to put in the DNA test sample.

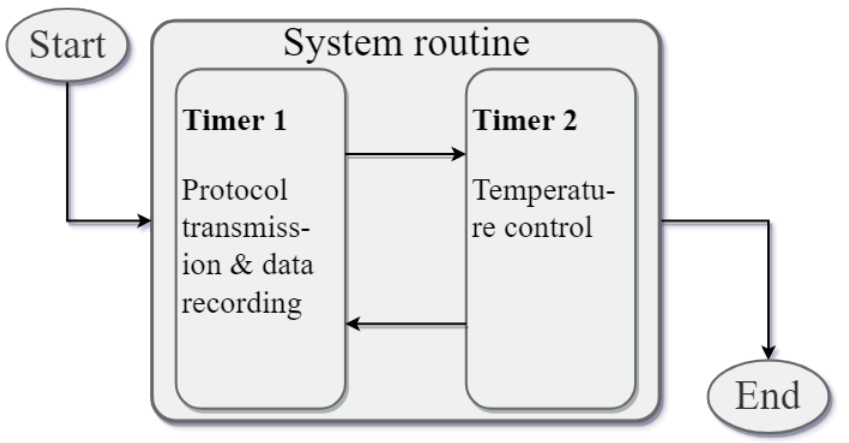

Fig. 2. Overall PCR behavior.

temperature difference is maintained at both ends of conductive layers when current is applied (Fig. 3).

\subsection{Learning machine data}

We stored the data we created in the device in a specific space in order to obtain experimental data. The left unit in Fig. 4 shows a simplified representation of the description. The machine on the left is the experimental machine. Also, the accumulated data on the right is defined as "Input Data" because it is used for machine learning.

Here is a brief description of the data. Many files that have been accumulated in the course of many experiments are unprocessed and contain a lot of information such as measured 


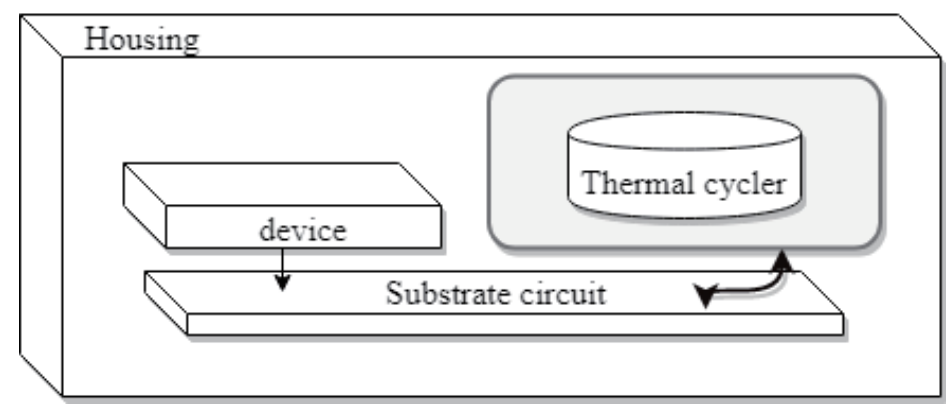

Fig. 3. Test thermal cycler design.

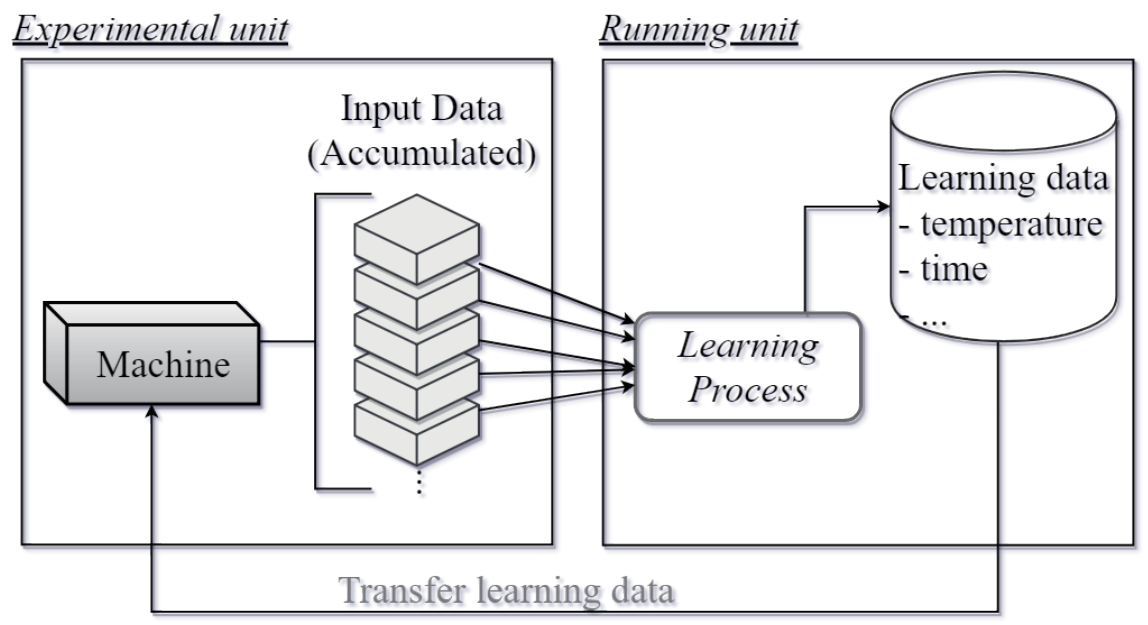

Fig. 4. Structure of data learning process.

temperature, time, and target temperature. However, we chose "measured temperature" and "time" as the data to be processed. Because Peltier modules slowly age with the repeated rise and fall of temperature, machine learning requires the elapsed time to reach the target temperature and whether or not the measured temperature reaches the target temperature.

\section{Results}

Initially, from PCR experiments, where device aging markedly affects the experiment, we accumulated experimental data of the device while continuing the experiment. The data were processed by determining the most suitable factor for the prediction of the degree of machine aging. To use the processed data, we selected the appropriate learning model. Typically, we used a simple but intuitive deep learning model, such as Keras, and obtained the following results.

Figure 5 shows a graph obtained by converting the data from the PCR experiment to a temperature cycle. It can be seen that the cycle becomes longer and more unstable over time. 

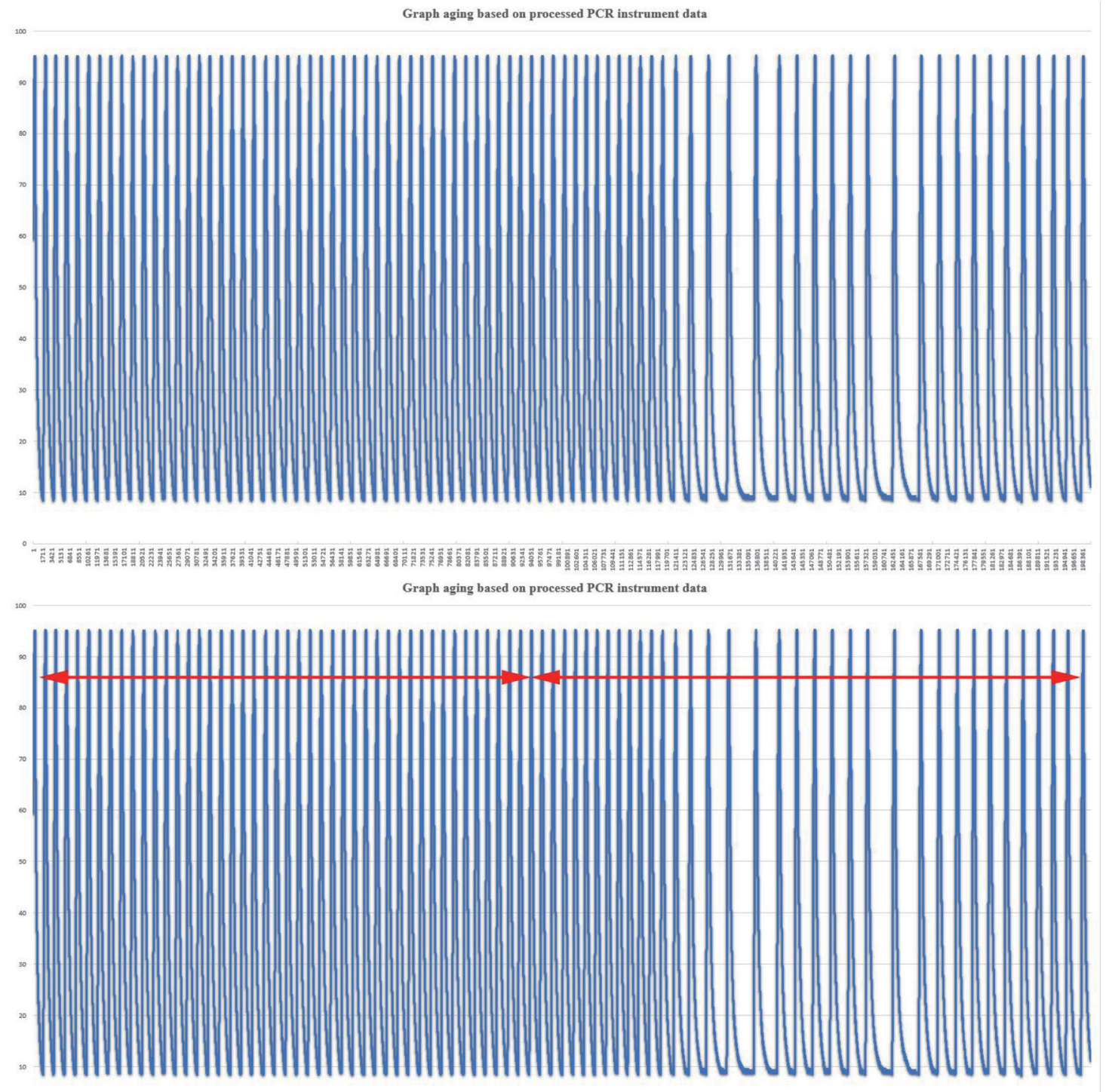

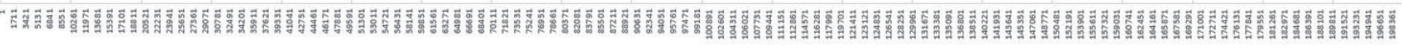

Fig. 5. (Color online) Graph of aging prepared using processed PCR instrument data.

From this figure, we obtain sample data of the aging of the instrument. To analyze the sample data more accurately, the following extraction was performed.

In Fig. 6, we observe a more detailed period difference, and during learning, we used experimental results as an indicator of normal and aging data. In addition, when testing was conducted using the data already learned again as a sample, devices predicted to age sometimes succeeded. Thus, we will accumulate more data to obtain better results. 
(a)

(b)

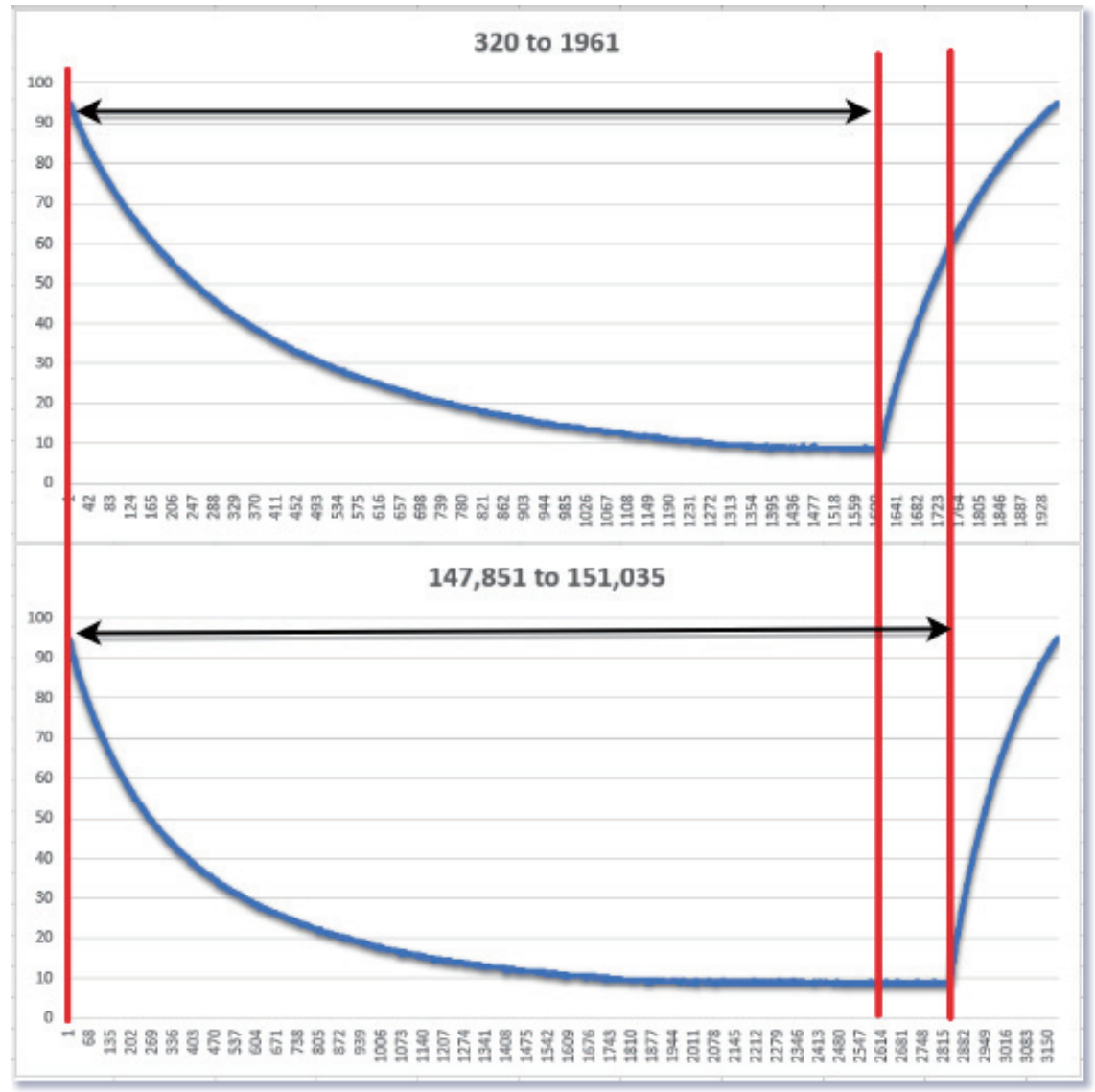

Fig. 6. (Color online) (a) 320 to 1961 sample data and (b) 147851 to 151035 sample data.

\section{Conclusions}

In this study, we succeeded in measuring the degree of senescence using variables that have important effects on the instrument, and by processing the data, we confirmed the degree of senescence of the instrument. However, in spite of using data learning, it is expected that sensitive machine learning data in self-learning will require a learning model for a larger amount of data than that used in this study.

\section{Acknowledgments}

This research was supported by The Leading Human Resource Training Program of Regional Neo Industry through the National Research Foundation of Korea (NRF) funded by the Ministry of Science, ICT and Future Planning (NRF-2016H1D5A1909654).

\section{References}

1 L. A. Geddes: Medical Device Accidents and Illustrative Cases (Lawyers \& Judges Publishing, Tuscon, AZ, USA, 2002)

2 H. A. Erlich: J. Clin. Immunol. 9 (1989) 437. https://doi.org/10.1007/BF00918012

3 C.-Y. Park, J.-D. Kim, Y.-S. Kim, H.-J. Song, J.-M. Kim, and J. Kim: Int. J. Multimedia Ubiquitous Eng. 7 (2012) 389.

4 J.-S. Hwang, J.-D. Kim, Y.-S. Kim, H.-J. Song, and C.-Y. Park: Sens. Mater. 30 (2018) 397. 
5 D.-J. Lee, J.-D. Kim, Y.-S. Kim, H.-J. Song, and C.-Y. Park: Biomed. Eng. Online 17 (2018) 150.

6 J.-S. Hwang, J.-D. Kim, Y.-S. Kim, H.-J. Song, and C.-Y. Park: Biomed. Eng. Online 17 (2018) 156.

7 M. I. Jordan and T. M. Mitchell: Science 349 (2015) 255.

8 F. Chollet: https://keras.io.

9 J. K. Nelson, S. Azizi-Ghannad, and H. Li: IEEE Trans. Dielectr. Electr. Insul. 7 (2000) 773. https://doi. org/10.1109/94.891988

10 M.-S. Lee. C.-Y. Park, Y.-S. Kim, H.-J. Song, and J.-D. Kim: Adv. Sci. Technol. Lett. 143 (2017) 113.

11 T. Harman, J. Cahn, and M. Logan: J. Appl. Phys. 30 (1959) 1351. https://doi.org/10.1063/1.1735334

12 J. Kim, J. Kim, and G. Lee: Proc. SPIE, Microfluidics, BioMEMS, and Medical Microsystems IX 7929 (2011) $79290 Y$.

\section{About the Authors}

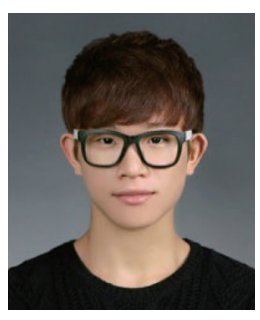

Jeong-Uk Park graduated from Hallym University and is now with the graduate school of Hallym University. He is also a researcher at Bio-IT Research Center.

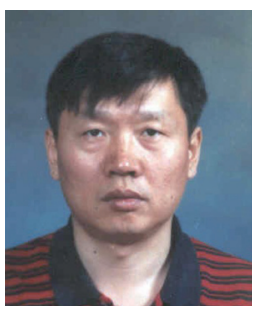

Jong-Dae Kim received his M.S. and Ph.D. degrees in electrical engineering from Korea Advanced Institute of Science and Technology, Seoul, Korea, in 1984 and 1990, respectively. He worked for Samsung Electronics from 1988 to 2000 as an electrical engineer. He is now a professor in the Department of Ubiquitous Computing, Hallym University. His recent interests focus on biomedical system and bioinformatics.

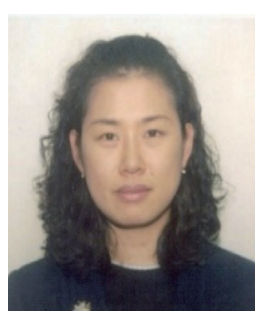

Hye-Jeong Song received his Ph.D. degree in computer engineering from Hallym University. He is a professor in the Department of Ubiquitous Computing, Hallym University. His recent interests focus on biomedical systems and bioinformatics.

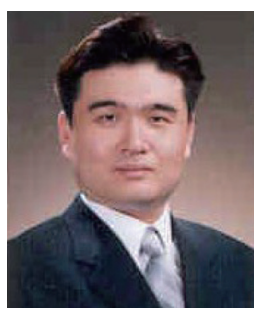

Yu-Seop Kim received his Ph.D. degree in computer engineering from Seoul National University. He is currently a professor in the Department of Ubiquitous Computing at Hallym University, South Korea. His research interests are in the areas of bioinformatics, computational intelligence, and natural language processing.

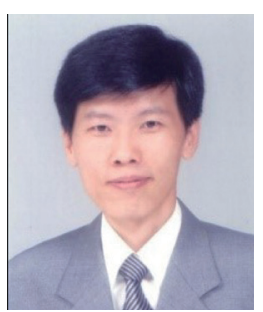

Chan-Young Park received his B.S. degree from Seoul National University and his M.S. and Ph.D. degrees from the Korea Advanced Institute of Science and Technology in 1989 and 1995, respectively. From 1991 to 1999, he worked for Samsung Electronics. He is currently a professor in the Department of Convergence Software at Hallym University. His research interests are in the areas of Bio-IT convergence and sensor networks. (cypark@hallym.ac.kr) 